\title{
Maternal high-fat diet triggers lipotoxicity in the fetal livers of nonhuman primates
}

\author{
Carrie E. McCurdy, ${ }^{1}$ Jacalyn M. Bishop, ${ }^{2}$ Sarah M. Williams, ${ }^{2}$ Bernadette E. Grayson, ${ }^{2}$
} M. Susan Smith, ${ }^{2}$ Jacob E. Friedman, ${ }^{1}$ and Kevin L. Grove ${ }^{2}$

\author{
1Department of Pediatrics, University of Colorado Denver, Aurora, Colorado, USA. 2Division of Neuroscience, \\ Oregon National Primate Research Center (ONPRC), Oregon Health and Science University, Beaverton, Oregon, USA.
}

\begin{abstract}
Maternal obesity is thought to increase the offspring's risk of juvenile obesity and metabolic diseases; however, the mechanism(s) whereby excess maternal nutrition affects fetal development remain poorly understood. Here, we investigated in nonhuman primates the effect of chronic high-fat diet (HFD) on the development of fetal metabolic systems. We found that fetal offspring from both lean and obese mothers chronically consuming a HFD had a 3-fold increase in liver triglycerides (TGs). In addition, fetal offspring from HFD-fed mothers (O-HFD) showed increased evidence of hepatic oxidative stress early in the third trimester, consistent with the development of nonalcoholic fatty liver disease (NAFLD). O-HFD animals also exhibited elevated hepatic expression of gluconeogenic enzymes and transcription factors. Furthermore, fetal glycerol levels were 2 -fold higher in O-HFD animals than in control fetal offspring and correlated with maternal levels. The increased fetal hepatic TG levels persisted at P180, concurrent with a 2-fold increase in percent body fat. Importantly, reversing the maternal HFD to a low-fat diet during a subsequent pregnancy improved fetal hepatic TG levels and partially normalized gluconeogenic enzyme expression, without changing maternal body weight. These results suggest that a developing fetus is highly vulnerable to excess lipids, independent of maternal diabetes and/or obesity, and that exposure to this may increase the risk of pediatric NAFLD.
\end{abstract}

\section{Introduction}

The prevalence of childhood obesity has increased dramatically in the United States over the past several decades. According to the most recent National Health and Nutrition Examination Survey (NHANES) data, almost one-third of children between 6 and 19 years of age are overweight, and $12 \%$ of infants are considered overweight $(1,2)$. This represents a 2 - to 3 -fold rise in childhood obesity since the initial NHANES data from 1971 to 1974 (3). Paralleling this disturbing trend is a dramatic rise in the diagnosis of type II diabetes and nonalcoholic fatty liver disease (NAFLD) in the juvenile population $(4,5)$. The increase in metabolic disorders is attributed primarily to calorically dense foods and decreased physical activity. However, prenatal metabolic events likely contribute to the increased risk of childhood metabolic disorders (6). Women with gestational diabetes (GDM) often have macrosomic babies that are at higher risk for developing metabolic diseases later in life (7-11). This increased risk in offspring of GDM mothers is independent of genetics, as children born to diabetic mothers are at significantly higher risk for future obesity and diabetes than are their siblings born prior to the onset of maternal diabetes

Authorship note: Carrie E. McCurdy and Jacalyn M. Bishop contributed equally to this work and are co-first authors.

Conflict of interest: The authors have declared that no conflict of interest exists.

Nonstandard abbreviations used: DEXA, dual energy X-ray absorptiometry; FBP1, fructose 1,6-bisphosphatase 1; G130, gestational day 130; GDM, gestational diabetes; G6P, glucose 6 phosphatase; GTT, glucose tolerance test; HFD, high-fat diet; HFREV, animals that were switched from HFD to normal chow 1-3 months before becoming pregnant in the fifth year; HFY4, animals pregnant in the fourth year of HFD feeding; HNE, 4-hyroxy-2-nonenal; HNF4 $\alpha$, hepatocyte nuclear factor $4 \alpha$; NAFLD, nonalcoholic fatty liver disease; NHP, nonhuman primate; O-, offspring; 8-OH-DG, 8-hydroxy-deoxyguanisine; PCK1, phosphoenolpyruvate carboxykinase; PGC1A, PPAR $\gamma$ coactivator $1 \alpha$; -R, HFD resistant; -S, HFD sensitive; TG, triglyceride; WAT, white adipose tissue.

Citation for this article: J. Clin. Invest. 119:323-335 (2009). doi:10.1172/JCI32661.
$(12,13)$. In addition, results of studies in numerous animal models (rats, mice, and sheep) support the role of altered nutritional status during pregnancy and the early postnatal period in predisposing offspring to the development of metabolic disorders (14-24). However, whereas most of these studies have focused on maternal nutrient restriction, the vast majority of our population is experiencing an environment of nutrient excess, and the increase in maternal obesity is a new cause for concern.

While maternal hyperglycemia/hyperinsulinemia contributes to increased fetal growth and the development of metabolic disorders in offspring (25), recent work suggests that maternal pregravid weight and triglyceride (TG) levels may be a better correlate of excessive fetal growth (26-29). These findings imply that maternal hyperlipidemia alone could contribute to the development of metabolic disorders in offspring. Outside of pregnancy, hyperlipidemia is often associated with hepatic and skeletal muscle fat accumulation, leading to system-wide insulin resistance. Compromised insulin signaling cascades have been implicated in this process (30, 31). There is increasing interest in the hypothesis that exposure to maternal obesity during pregnancy, in the absence of diabetes, may have adverse lifelong consequences in the offspring (32-35); however, the underlying mechanisms by which fetal metabolic systems cope with excess fuels during intrauterine development remain relatively unexplored. One hypothesis for the potential adverse effects of excess fuels on fetal development may be a lack of white adipose tissue (WAT) in most species until relatively late in pregnancy. For example, in humans and nonhuman primates (NHPs), the development of WAT occurs during the third trimester $(36,37)$. It is well accepted that WAT is critical for storage of excess lipids and that a lack of WAT results in whole-body insulin resistance and susceptibility to fatty liver in adult humans (38-45).

To begin to address this issue, we used a NHP model to determine the effect of chronic maternal consumption of a high- 
Table 1

Nutrient specifications of control diet and HFD

\begin{tabular}{|c|c|c|c|c|}
\hline & \multicolumn{2}{|c|}{$\begin{array}{l}\text { Physical } \\
\text { composition }\end{array}$} & \multicolumn{2}{|c|}{$\begin{array}{c}\text { Metabolizable } \\
\text { energy }\end{array}$} \\
\hline & Control & HFD & Control & HFD \\
\hline Protein $(\%)$ & 20.6 & 17.1 & 26.8 & 16.1 \\
\hline Carbohydrate (\%) & 70.8 & 55.5 & 58.5 & 52.3 \\
\hline Fat $(\%)$ & 5.5 & 14.9 & 14.7 & 31.6 \\
\hline Saturated (\%) & 1.69 & 2.46 & & \\
\hline Monounsaturated (\%) & 1.65 & 2.53 & & \\
\hline Polyunsaturated (\%) & 2.58 & 3.05 & & \\
\hline Metabolizable energy $(\mathrm{kcal} / \mathrm{g}$ ) & & & 2.87 & 3.80 \\
\hline Cholesterol (ppm) & & & 83 & 946 \\
\hline
\end{tabular}

Nutrients are as reported by the manufacturers (see Methods).

calorie, high-fat diet (HFD) on the development of metabolic systems in the fetal offspring. We demonstrate that only a portion of the adult female monkeys chronically consuming the HFD became obese and insulin resistant. However, regardless of maternal obesity and insulin resistance, all fetal offspring of HFD mothers (O-HFD animals) examined during the early third trimester showed signs of NAFLD, including hepatic inflammation, oxidative stress and/or damage, TG accumulation, and premature gluconeogenic gene activation. The elevation in liver TG levels persisted in the O-HFD group compared with control offspring measured at P30 and P180. The persistence in hepatic TGs in the postnatal period was accompanied by a 2 -fold increase in percent body fat of O-HFD animals compared with control offspring, which suggested that the fetal phenotype did not reverse with development of WAT at the end of the third trimester. Importantly, we also found that switching the diet of mothers fed the HFD for 4 years to the control low-fat diet during a subsequent pregnancy reduced many of the abnormalities in the fetal liver, even though some mothers remained obese and insulin resistant. Our findings strongly implicate that exposure to excess maternal lipids during early fetal development may increase the risk for NAFLD and metabolic diseases during postnatal life.

\section{Results}

HFD feeding induces obesity and insulin resistance in adult nonpregnant female macaques. Adult female Japanese macaques, starting at 5-7 years of age, were placed on either a control diet or the HFD (Table 1) for 2-4 years. Because the animals were socially housed, individual food intake, activity, and energy expenditure could not be determined. Animals fed the HFD were divided into 2 groups based on body weight gain and insulin resistance. Animals were considered sensitive to the HFD (HFD-S) if they displayed increased AUC for insulin secretion at least 2 SD above the mean of the controls during i.v. glucose tolerance tests (GTTs) by the end of the first year on the diet. While there was an apparent decrease in insulin sensitivity by the end of the first year, most animals did not display significant weight gain until the end of the second year on the HFD. In contrast, animals resistant to the HFD (HFD-R) displayed no significant weight gain and had normal insulin secretion during the i.v. GTT throughout the 4 years on the diet. Approximately $60 \%$ of the animals were HFD-S. The metabolic phenotype of all of the adult animals that became pregnant during these studies is summarized in Supplemental Table 1 (prepregnancy data; supplemental material available online with this article; doi:10.1172/JCI32661DS1) and Table 2 (maternal third trimester data); the HFD group includes animals fed the HFD for 2-4 years. As a group, the HFD-S nonpregnant animals had significantly increased body weight and significantly increased insulin secretion during i.v. GTT compared with the control group; however, basal fasting insulin levels were not different. Neither fasting glucose nor glucose clearance (glucose AUC) during the i.v. GTT was different between the 3 groups prior to pregnancy. HFD-S animals also had increased leptin and glycerol levels; however, the difference was only significant in pregnant HFD-S animals versus controls because of animal-to-animal variability. The HFD-R animals did not show significant differences in any of the measures compared with the age-matched, control diet-fed group.

By the completion of these studies, a subset of animals had been on the HFD for 4 years; the basic phenotype in these 7 animals is summarized in Supplemental Table 1. By the fourth year on the HFD, the HFD-S group had a $35 \%$ increase in body weight, a greater than 3-fold increase in insulin AUC during GTT, a more than 5-fold increase in leptin levels, and a near doubling in body fat as determined by dual energy X-ray absorptiometry (DEXA; Supplemental

\section{Table 2}

Maternal third-trimester data

\begin{tabular}{|c|c|c|c|c|c|c|c|}
\hline & Control & HFD-R & HFD-S & HFY4-R & HFY4-S & HFREV-R & HFREV-S \\
\hline$n$ & 17 & 8 & 10 & 3 & 4 & 3 & 4 \\
\hline Weight (kg) & $8.9 \pm 0.4$ & $9.5 \pm 0.4$ & $10.6 \pm 0.5^{A}$ & $8.50 \pm 0.45$ & $11.28 \pm 1.09^{B}$ & $9.68 \pm 0.69$ & $12.51 \pm 0.93^{C}$ \\
\hline Insulin $(\mu \mathrm{U} / \mathrm{ml})$ & $24.9 \pm 5.1$ & $32.1 \pm 14.0$ & $28.3 \pm 4.6$ & $40.0 \pm 26.2$ & $31.4 \pm 8.2$ & $11.9 \pm 2.7$ & $20.6 \pm 4.0$ \\
\hline Glucose (mg/dl) & $47.0 \pm 3.7$ & $39.4 \pm 3.7$ & $47.1 \pm 4.5$ & $43.0 \pm 3.5$ & $42.8 \pm 4.2$ & $46.0 \pm 1.7$ & $41.3 \pm 7.0$ \\
\hline Insulin AUC & $3,882 \pm 692$ & $4,103 \pm 766$ & $8,009 \pm 1,545^{A}$ & $4,541 \pm 1,268$ & $11,300 \pm 2,664^{D}$ & $2,430 \pm 785$ & $8,375 \pm 3,139 c$ \\
\hline Glucose AUC & $4,053 \pm 170$ & $3,461 \pm 310$ & $3,914 \pm 280$ & $3,663 \pm 545$ & $4,283 \pm 297$ & $3,875 \pm 523$ & $4,894 \pm 692$ \\
\hline Leptin (ng/ml) & $25.4 \pm 4.9$ & $55.1 \pm 11.4$ & $54.7 \pm 10.0^{A}$ & $65.0 \pm 28.3$ & $54.2 \pm 16.5$ & $20.9 \pm 14.4$ & $24.4 \pm 14.0$ \\
\hline Total TGs (mg/ml) & $0.55 \pm 0.07$ & $0.63 \pm 0.09$ & $0.83 \pm 0.14$ & $0.82 \pm 0.14$ & $0.80 \pm 0.29$ & $0.55 \pm 0.08$ & $0.43 \pm 0.14$ \\
\hline True TGs (mg/ml) & $0.56 \pm 0.05$ & $0.64 \pm 0.07$ & $0.73 \pm 0.11$ & $0.76 \pm 0.12$ & $0.74 \pm 0.21$ & $0.59 \pm 0.07$ & $0.49 \pm 0.11$ \\
\hline Glycerol (mg/ml) & $0.15 \pm 0.03$ & $0.11 \pm 0.02$ & $0.26 \pm 0.06^{A}$ & $0.16 \pm 0.03$ & $0.26 \pm 0.07$ & $0.13 \pm 0.03$ & $0.11 \pm 0.03$ \\
\hline
\end{tabular}

Values (mean \pm SEM) are only from adult animals that became pregnant. Animals in the HFD group had been fed HFD for $2-4$ years at the time of data collection. The control group represents a separate group of age-matched animals maintained on a standard chow diet. AP $<0.05$ among control, HFD-R, and HFD-S (ANOVA with Tukey's post-hoc analysis). ${ }^{\mathrm{B} P}<0.05$ versus HFY4-R (2-tailed Student's $t$ test). ${ }^{C} P<0.05$, combined HFY4 versus combined HFREV groups (paired 2-tailed Student's $t$ test). ${ }^{D} P<0.01$ versus HFY4-R (2-tailed Student's $t$ test). 

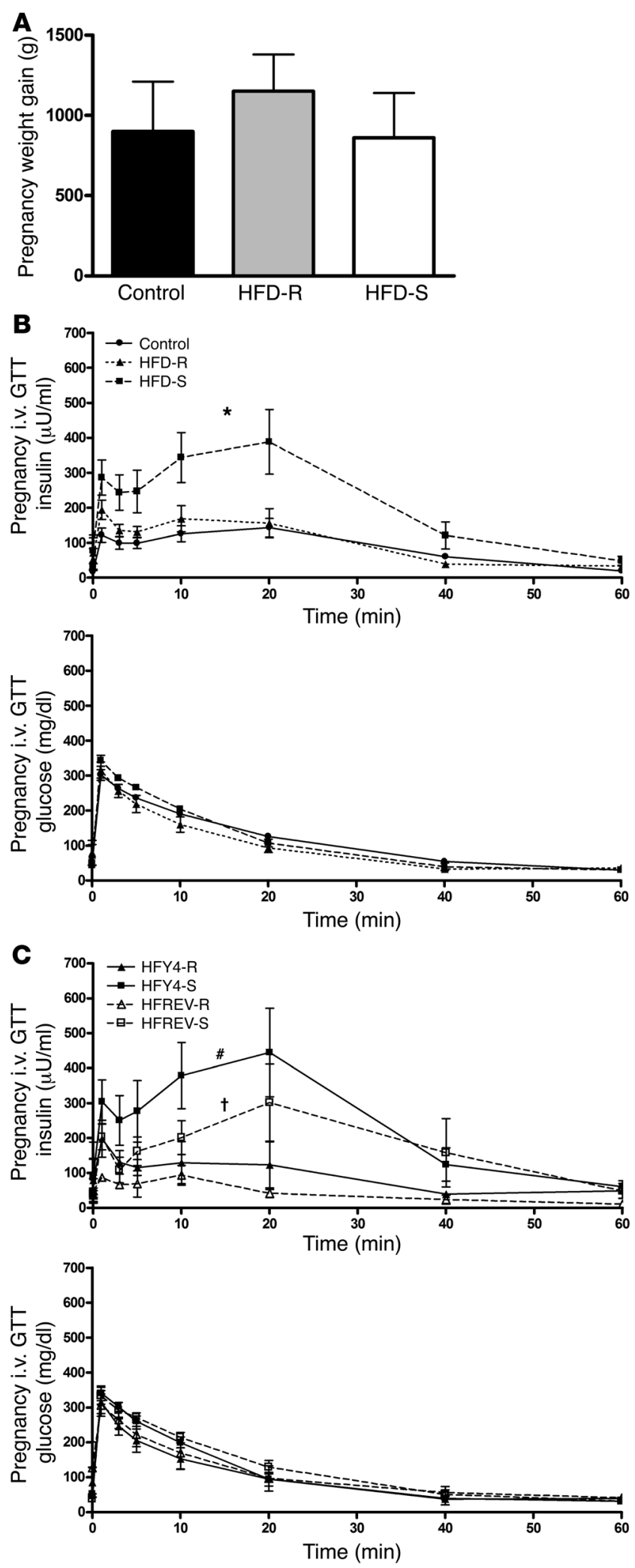

Table 1) compared with the control and HFD-R groups. Fasting glucose levels were not significantly elevated in either the HFD-S group or the HFD-R group, even by the end of the fourth year (data not shown), although the HFD-S group had an increase in

\section{Figure 1}

HFD-fed pregnant female macaques display hyperinsulinemia and hyperglycerolemia independent of weight gain. All data in this figure were obtained from animals at G130. (A) Weight gain during pregnancy was similar in all 3 groups. Data were analyzed using 1-way ANOVA with Bonferroni post-hoc analysis. (B) Insulin secretion and glucose clearance during i.v. GTT for control, HFD-R, and HFD-S groups. The HFD groups are from animals fed HFD for 2-4 years and were obtained during the early third trimester. ${ }^{*} P<0.05$ versus control. (A and B) $n=17$ (control); 10 (HFD-S); 8 (HFD-R). (C) Insulin secretion and glucose clearance during i.v. GTT for HFY4 and HFREV groups. $\# P<0.05$ versus HFY4-R; ${ }^{\dagger} P<0.05$ versus HFY4-S, 2-tailed Student's $t$ test. $n=3$ (HFY4-R and HFREV-R); 4 (HFY4-S and HFREV-S).

homeostasis model assessment of insulin resistance (HOMAIR, see Methods and refs. 46, 47; control, $2.53 \pm 0.50$; HFD-R, $2.81 \pm 0.70$; HFD-S, $7.87 \pm 1.50 ; P<0.05$, HFD-S versus control). The HFD-R animals retained body weight and metabolic phenotype similar to that of the controls throughout the entire 4 years.

Chronic consumption of a HFD provokes insulin resistance and byperleptinemia in pregnant macaques. In the present study, all animals had similar weight gain during pregnancy (Figure 1A). Additionally, all animals displayed pregnancy-induced fasting hyperleptinemia (Table 2 and Supplemental Table 1), which was most evident during the third trimester. There was a greater increase in leptin levels in both HFD-R and HFD-S animals during pregnancy; however, only the increase in HFD-S was significant (Table 2). Insulin AUCs during third-trimester i.v. GTT significantly increased in HFD-S animals compared with control and HFD-R animals (Figure 1B and Table 2). There was no difference in insulin AUC between HFD-R and control groups. Fasting glucose or glucose AUC, diagnostics for GDM, were not different between any of the groups. Finally, while levels of serum free fatty acids and TGs were not significantly elevated in the HFD groups, glycerol levels during the third trimester were significantly elevated only in HFD-S animals (Table 2 and Supplemental Table 1). All groups consisted of animals undergoing their first, second, or third pregnancies.

Table 2 summarizes the phenotypic data from 7 animals that were maintained on the HFD through their fourth-year pregnancy (HFY4) and were switched back to the control diet approximately 1-3 months prior to becoming pregnant in the fifth year (HFREV). HFREV animals did not show any decrease in body weight; in fact, the third-trimester body weights of these animals were higher than those of the HFY4 group (Table 2). In spite of the increased body weight, the HFREV group did have slightly, but significantly, lower insulin AUC during the i.v. GTT (Table 2 and Figure 1C). Fasting insulin, leptin, and glycerol levels were also lower; however, the differences were not significant (Table 2).

Chronic maternal HFD feeding impacts fetal growth and results in earlyonset obesity. In our evaluation of the fetuses, we focused on gestational day 130 (G130), during the early third trimester. This period was chosen to determine the metabolic consequences in the fetus prior to and during important developmental stages in multiple organs. Our hypothesis was that the fetus would be susceptible to lipotoxicity prior to the development of WAT, which occurs later in the third trimester $(36,37)$. Third-trimester fetuses were collected by cesarean section from mothers chronically consuming the HFD for $2-4$ years $(n=6$ [2 yr]; 4 [3 yr]; 8 [4 yr]); 8 were offspring from HFD-R mothers (O-HFD-R), and 10 were from HFD-S mothers (O-HFD-S). Control fetal offspring $(n=17)$ were collected from 
Table 3

Fetal third-trimester data

$\begin{array}{lccccc} & \text { Control } & \text { O-HFD-R } & \text { O-HFD-S } & \text { 0-HFREV-R } & \text { 0-HFREV-S } \\ n & 17 & 8 & 10 & 3 & 4 \\ \text { Weight }(\mathrm{g}) & 355.6 \pm 6.43 & 321.1 \pm 10.9^{A} & 330.8 \pm 8.8^{A} & 363.3 \pm 13.6 & 354.3 \pm 10.8 \\ \text { Liver weight }(\mathrm{g}) & 9.57 \pm 0.19 & 9.38 \pm 0.39 & 10.19 \pm 0.46 & 9.76 \pm 1.12 & 10.41 \pm 0.34 \\ \text { Retro pad weight }(\mathrm{mg}) & 1,19.1 \pm 21.2 & 137.3 \pm 51.0 & 126.3 \pm 16.3 & 106.7 \pm 13.3 & 101.3 \pm 13.9 \\ \text { Insulin }(\mu \mathrm{U} / \mathrm{ml}) & 5.28 \pm 0.80 & 3.55 \pm 0.85 & 6.21 \pm 1.08 & 6.82 \pm 0.17 & 6.33 \pm 1.87 \\ \text { Glucose }(\mathrm{mg} / \mathrm{dl}) & 115.1 \pm 10.8 & 105.7 \pm 14.2 & 90.7 \pm 8.2 & 117.3 \pm 4.8 & 128.8 \pm 32.7 \\ \text { NEFAs }(\mathrm{mEg} / \mathrm{l}) & 0.11 \pm 0.02 & 0.13 \pm 0.03 & 0.13 \pm 0.02 & 0.15 \pm 0.01 & 0.13 \pm 0.03 \\ \text { Leptin }(\mathrm{ng} / \mathrm{ml}) & 0.54 \pm 0.02 & 0.54 \pm 0.03 & 0.54 \pm 0.03 & <0.50 & <0.50 \\ \text { Total TGs }(\mathrm{mg} / \mathrm{ml}) & 0.22 \pm 0.02 & 0.32 \pm 0.05^{\mathrm{A}} & 0.40 \pm 0.06^{\mathrm{A}, \mathrm{B}} & 0.24 \pm 0.08 & 0.238 \pm 0.07 \\ \text { True TGs }(\mathrm{mg} / \mathrm{ml}) & 0.31 \pm 0.01 & 0.36 \pm 0.03 & 0.39 \pm 0.04 & 0.31 \pm 0.05 & 0.30 \pm 0.05 \\ \text { Glycerol }(\mathrm{mg} / \mathrm{ml}) & 0.09 \pm 0.01 & 0.15 \pm 0.04^{\mathrm{A}} & 0.18 \pm 0.03^{\mathrm{A}, \mathrm{B}} & 0.07 \pm 0.02 & 0.08 \pm 0.03\end{array}$

Values are mean \pm SEM. Control offspring were from age-matched animals ( 9 female, 8 male). The combined O-HFD group had 10 females and 8 males; the combined O-HFREV group had 5 females and 2 males. All offspring were singleton births. Data from the O-HFREV group were only compared with those from controls obtained during the same breeding season. NEFAs, nonesterified fatty acids. ${ }^{A} P<0.05$, combined O-HFD group versus control. ${ }^{\mathrm{B} P}<0.05, \mathrm{O}-\mathrm{HFD}-\mathrm{S}$ versus control (ANOVA).
O-HFD-S and O-HFD-R groups compared with control offspring (Table 3). Fetal glycerol levels were also significantly correlated with maternal glycerol levels $\left(r^{2}\right.$, 0.143 ; $P<0.03)$; however, none of the other parameters tested were significantly correlated, including insulin, leptin, glucose, and TGs. To determine the relative impact of circulating cytokines, we used the Proteome Profiler Cytokine Panel (see Methods) to determine plasma cytokine levels. Of the 36 cytokines probed, 16 were significantly upregulated as a result of maternal HFD consumption $(P \leq 0.05)$, with 5 more showing a near-significant increase (Supplemental Table 2).

To determine whether maternal age-matched females. There was no significant effect of age or time on the diet (i.e., between 2 and 4 years) for any of the outcomes measured. All of the groups were balanced with respect to fetal gender and maternal diet sensitivity (Table 3). With this cohort, there was no evidence of gender-specific effects.

As a group, the O-HFD (combined O-HFD-R and O-HFD-S) animals weighed $10 \%$ less than did control fetal offspring (Table 3 ). This decreased body weight was evident for both O-HFD-R and O-HFD-S groups, but the decrease was only significant when the groups were combined and compared with controls by 2 -tailed Student's $t$ test. This decrease was likely due to decreased lean body mass, because very little WAT was evident in G130 fetuses (Table 3 ), and the fat pad weight was less than $0.5 \%$ of total body mass at this gestational age. This conclusion was supported by our finding of nearly undetectable levels of leptin in all G130 fetuses (Table 3), for which the detection limits of the assay are $0.5 \mathrm{ng} / \mathrm{ml}$. This is consistent with human fetuses, in which WAT development occurs during the third trimester and circulating leptin levels are low until the mid-third trimester $(36,37)$. The low leptin levels observed in the third-trimester fetus suggest that little or no maternal leptin crosses the placenta. No differences were found in fetal serum insulin or free fatty acid levels among the groups (Table 3 ). Fetal total TG and glycerol levels were significantly higher in both

\section{Figure 2}

Maternal HFD feeding during pregnancy and the perinatal period increases offspring adiposity and liver TG levels. (A) Body weights of O-HFD and control offspring at P30, P90, and P180. There was no statistical difference between the groups at any age. (B) Whole body fat composition, as determined by DEXA analysis, at all 3 ages in both groups. (C) Liver TG levels in O-HFD and control offspring at $\mathrm{P} 30$ and P180. For TG analysis of P30 offspring, $n=4$ for both control and O-HFD (2 male and 2 female). Liver samples were obtained from these offspring at the time of termination. A separate group of animals was used to obtain the serial data in $\mathbf{A}$ and $\mathbf{B}$ and the P180 liver TGs in C. The control group consisted of 4 animals (2 male and 2 female), while the O-HFD group consisted of 7 animals ( 4 male and 3 female). Liver samples were obtained from these animals by biopsies obtained through laparoscopic surgery. ${ }^{*} P<0.05$ versus respective control, 2-tailed Student's $t$ test.
HFD and/or metabolic phenotype affected growth and adiposity that persisted into the postnatal period, a subset of pregnancies was carried to full term, and a cohort of control offspring and O-HFD animals ( $n=4$ per group) was investigated at P30. The P30 O-HFD group had slightly higher body weights (control, $700 \pm 34 \mathrm{~g}$; O-HFD, $797 \pm 26 \mathrm{~g}$ ) and increased leptin levels (control, $1.84 \pm 0.05 \mathrm{ng} / \mathrm{ml}$;

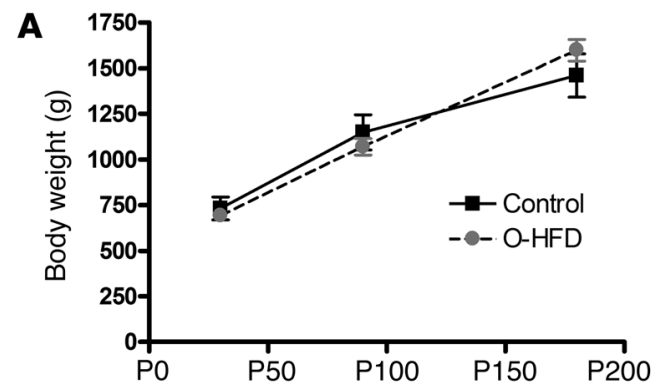

B
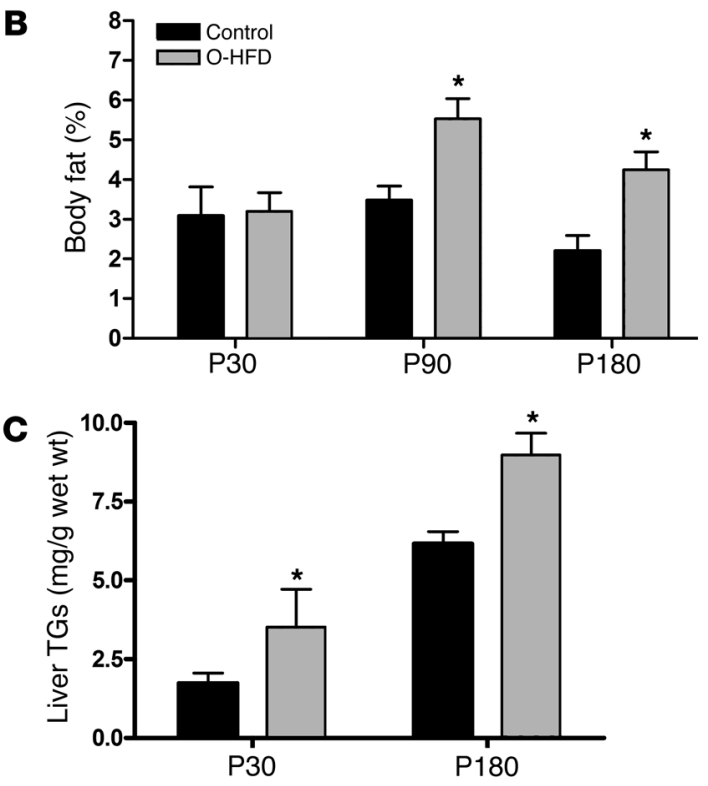

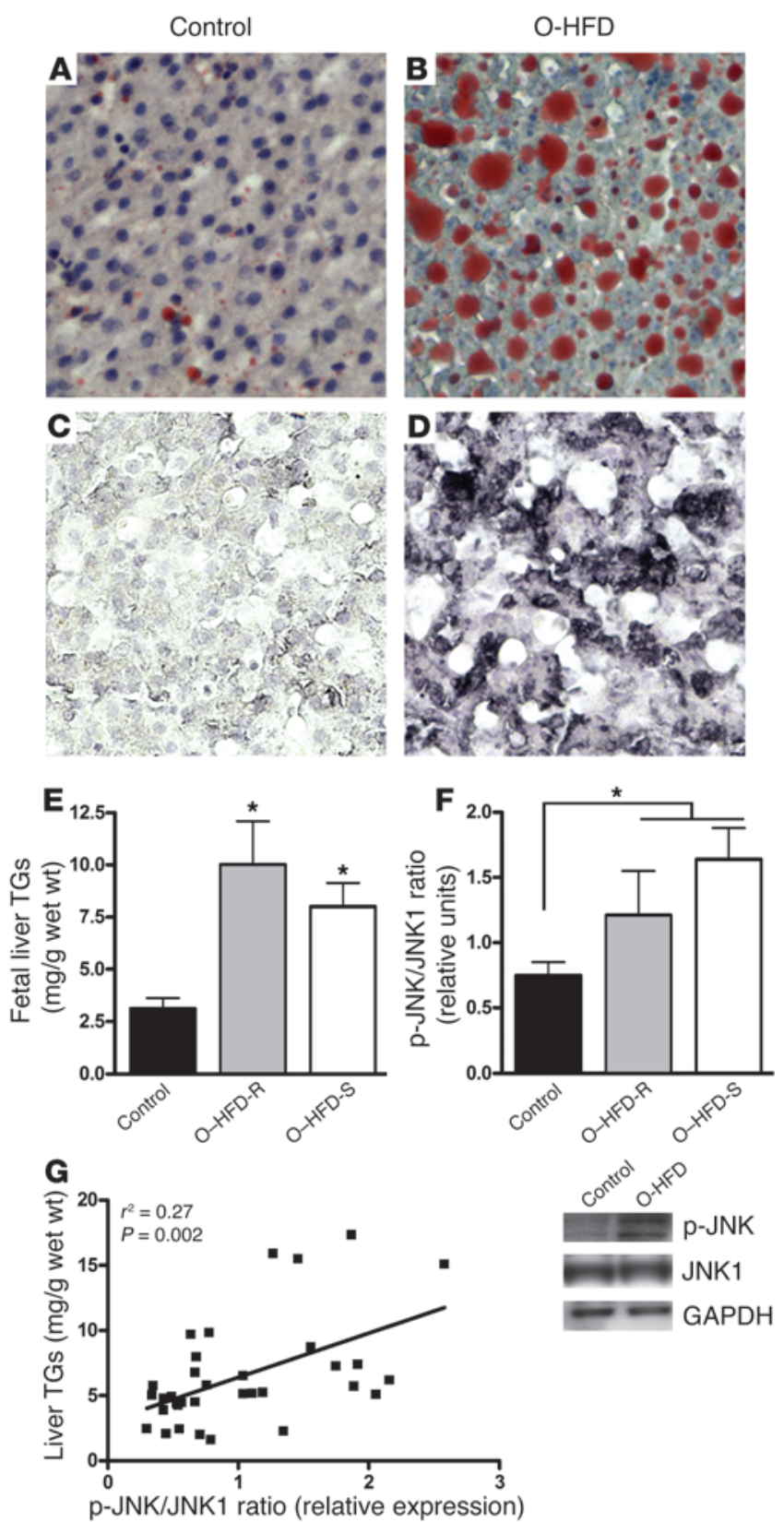

O-HFD, $2.16 \pm 0.1 \mathrm{ng} / \mathrm{ml}$ ), but the differences were not significant. These P30 O-HFD animals were similar in adiposity compared with control offspring based on DEXA analysis. These data suggest that O-HFD animals transitioned from $10 \%$ smaller in utero to normal body weight at P30.

A second cohort of pregnancies and the offspring was investigated for changes in body composition at P30, P90, and P180, and liver biopsies were obtained at the latest age. While body weights were not different at any age, there was a trend toward an increase by P180 (Figure 2A). However, the animals' percent body fat, as determined by DEXA, nearly doubled in the O-HFD group at P90 and P180 (Figure 2B). These animals were not terminated at P180, so further verification of body fat was not possible.

Chronic maternal HFD feeding triggers fatty liver and oxidative stress in the fetal liver that persists into the postnatal period. There is limited

\section{Figure 3}

Maternal HFD feeding leads to lipid accumulation and activation of oxidative stress in the fetal liver. (A-D) Oil Red $O$ staining (A and $\mathbf{B})$ and HNE staining ( $\mathbf{C}$ and $\mathbf{D})$ in the fetal livers of controls ( $\mathbf{A}$ and $\mathbf{C}$ ) and O-HFD animals (B and D). Original magnification, $\times 20$. (E) Fetal liver TGs were significantly elevated in both O-HFD-R and O-HFD-S groups. ${ }^{*} P<0.05$ versus control (1-way ANOVA with Bonferroni post-hoc analysis). (F) As a group, O-HFD animals had a significant increase in the ratio of $p-J N K 1$ to total JNK1 levels. Representative Western blots are also shown. ${ }^{*} P<0.05$ (2-tailed Student's $t$ test). (G) There was a significant correlation between TG levels and the ratio of p-JNK1 to total JNK1 in the fetal livers. $n=6$ (O-HFD-R); 9 (control and O-HFD-S).

capacity for de novo lipogenesis in the fetus, which suggests that the precursors for fetal fat accretion are primarily supplied transplacentally and consist of maternal substrates derived from lipids rather than from glucose (48-51). The significantly elevated plasma glycerol we observed in O-HFD fetuses is suggestive of increased maternal lipolysis. Figure 3 shows Oil Red O staining for lipids in the fetal liver of control offspring (Figure $3 \mathrm{~A}$ ) and O-HFD-S animals (Figure 3B). This histological analysis showed a dramatic increase in Oil Red O staining in the O-HFD group, whereas all 15 fetal offspring qualitatively displayed increased staining for lipids. Furthermore, liver TG levels were significantly increased in both O-HFD-S and O-HFD-R groups compared with control offspring (Figure 3E).

It is well known that chronic exposure to a HFD in adult life causes oxidative stress and/or damage in the liver (52-55). To determine whether exposure to a maternal HFD caused oxidative stress or damage in the fetal liver, we used qualitative histological analysis with 2 markers: 8-hydroxy-deoxyguanisine (8-OH-DG), a nuclear marker; and 4-hyroxy-2-nonenal (HNE), a cytoplasmic marker $(56,57)$. All samples from the O-HFD animals showed either a modest or a high level of 8-OH-DG (data not shown) and HNE staining (Figure 3, C and D), while minimal staining was evident in samples from controls. Furthermore, immunoblot analysis showed an increase in P-JNK1, with no change in total JNK1 protein levels. While there was not a significant increase in the ratio of p-JNK1 to total JNK1 in the O-HFD-S or O-HFD-R groups compared with controls as determined by ANOVA, there was a significant increase in the O-HFD group as a whole compared with controls when using 2-tailed Student's $t$ test (Figure 3F). Furthermore, the changes in p-JNK1 levels correlated to liver TG levels (Figure $3 \mathrm{G}$ ). Therefore, our combined findings of increased liver TGs and markers of oxidative stress are consistent with O-HFD NHPs showing early signs of NAFLD.

To further investigate the persistence of the fatty liver into the postnatal period, liver samples were analyzed from P30 and P180 animals. Even though P30 O-HFD animals did not have increased body weight or percent body fat, their liver TG levels significantly increased, and this persisted in the O-HFD group at P180 (Figure $2 \mathrm{C}$ ). It should be noted that within the control group, there were 2 female and 2 male offspring; within the HFD group, there were 4 male and 3 female offspring, with a balance between HFD-S and HFD-R. Moreover, 1 animal was removed from this data set because of failure to thrive as a result of extremely low liver TGs (3.5 mg TG/g wet wt), while another was removed from the data set because of extremely high liver TGs (22.58 mg TG/g wet wt), which was 2 SD beyond the mean of the group. 
A
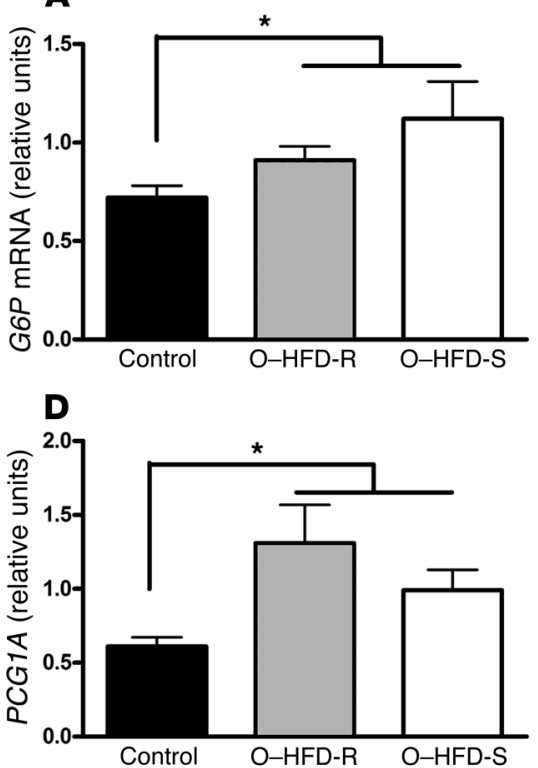

G

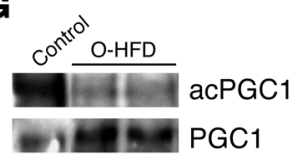

B

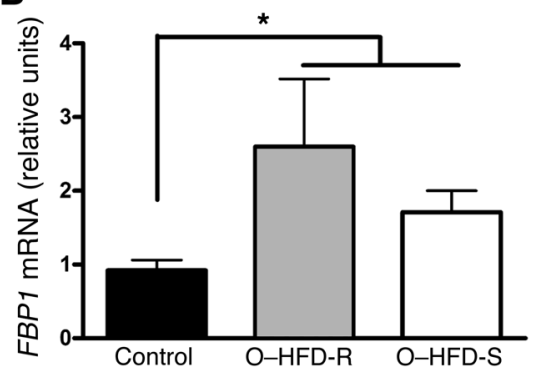

E
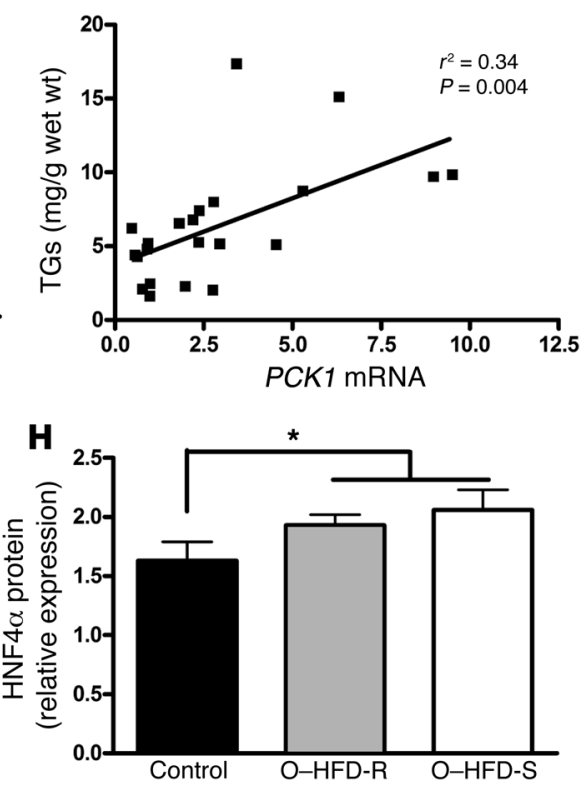

C

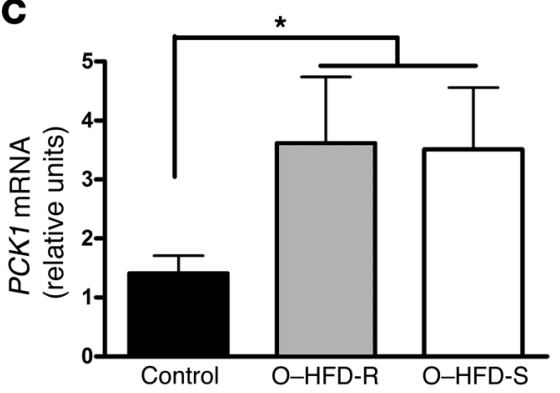

$\mathbf{F}$

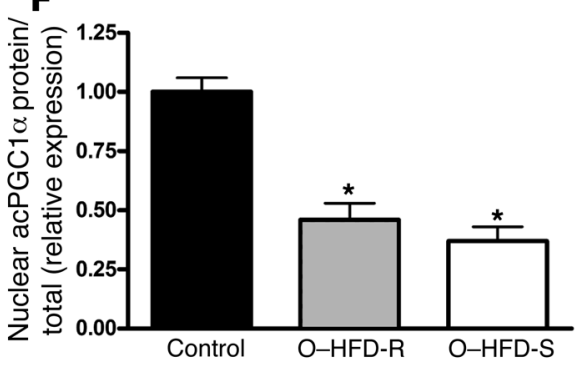

I

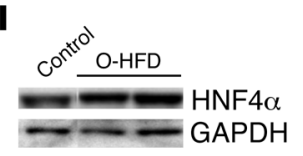

Figure 4

Maternal HFD feeding leads to increased expression of genes in the gluconeogenic pathway in the fetal liver. (A-D) As a group, O-HFD fetuses had significantly elevated levels of G6P (A), FBP1 (B), PCK1 (C), and PGC1A (D) mRNA in the liver. * $P<0.05$ (2-tailed Student's $t$ test). (E) $P C K 1$ mRNA expression significantly correlated with fetal liver TG levels. (F) The ratio of nuclear acetylated PGC1 $\alpha($ acPGC1 $\alpha)$ to total PGC1 $\alpha$ protein was significantly decreased in both O-HFD-R and O-HFD-S groups. ${ }^{*} P<0.05$ versus control (1-way ANOVA with Bonferroni post-hoc analysis). (G) Representative Western blot of acetylated PGC1 $\alpha$. (H) As a group, O-HFD animals had significantly elevated HNF4 $\alpha$ protein levels in the fetal liver. ${ }^{\star} P<0.05$ (2-tailed Student's $t$ test). (I) Representative Western blot of HNF4a. $n=6$ (O-HFD-R); 9 (control and O-HFD-S).

Chronic maternal HFD feeding reprograms the fetal hepatic gluconeogenic pathway. Hepatic gluconeogenesis is normally absent during fetal development, which is primarily attributed to the appearance of the rate-limiting enzyme phosphoenolpyruvate carboxykinase (PCK1) at the time of birth (58-62). Quantitative PCR demonstrated increased expression in 4 key enzymes in the gluconeogenic pathway in the O-HFD group: glucose 6 phosphatase (G6P), fructose 1,6-bisphosphatase 1 (FBP1), PPAR $\gamma$ coactivator $1 \alpha(P G C 1 A$, which encodes PGC1 $\alpha$ ), and PCK1 (2-tailed Student's $t$ test, Figure 4, A-D). The increase in these genes was 2- to 3-fold higher in both O-HFD-R and O-HFD-S groups compared with control offspring, but expression was lower than that found in the adult liver (Supplemental Figure 1). Not surprisingly, fetal liver TGs were significantly correlated with the increases in these genes, and the correlation with PCK1 mRNA was the strongest (Figure 4E).

As further confirmation of activation of the gluconeogenic genes, we performed immunoblot analysis on key transcriptional regulators in this pathway. While total tissue PGC1 $\alpha$ protein expression was not altered (Supplemental Table 3), there was a significant reduction in the acetylated form of PGC1 $\alpha$ in nuclear extracts of both O-HFD-S and O-HFD-R groups compared with control offspring (Figure 4, F and G), which is suggestive of an increase in PGC1 $\alpha$ activity in the O-HFD liver (63). PGC1 $\alpha$ also requires interaction with hepatocyte nuclear factor $4 \alpha(\mathrm{HNF} 4 \alpha)$ to induce gluconeogenic gene expression. As shown in Figure 4, $\mathrm{H}$ and I, HNF4 $\alpha$ protein levels were modestly, but significantly, increased in the liver of O-HFD animals compared with controls. No major impact was found on the levels of total or phosphorylated FOXO1, CCAAT/ enhancer-binding protein $\beta$, and phosphorylated or total cAMPresponsive element-binding protein (Supplemental Table 3). Taken together, these results suggest that HFD feeding induced a premature gluconeogenic gene expression program in fetal liver associated with posttranslational modification of PGC1 $\alpha$ and increased HNF $4 \alpha$. In the absence of fetal hyperglycemia or hyperinsulinemia, however, hepatic glucose production may be limited by the availability of gluconeogenic precursors, such as lactate and certain amino acids, at this stage of development (58). Interestingly, immunoblot analysis failed to detect changes in the protein 
A

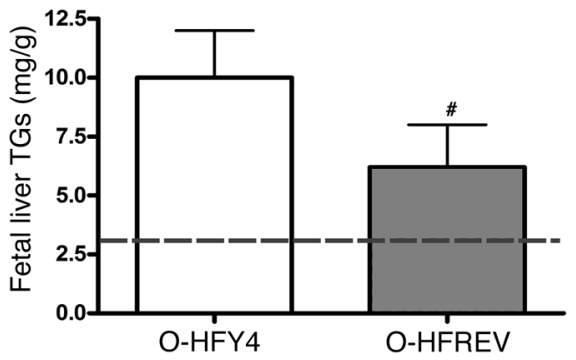

B

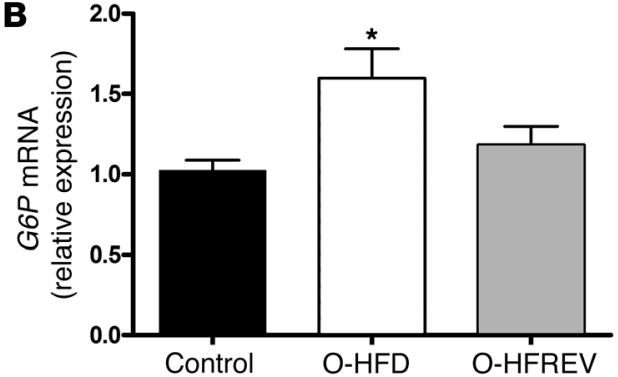

D

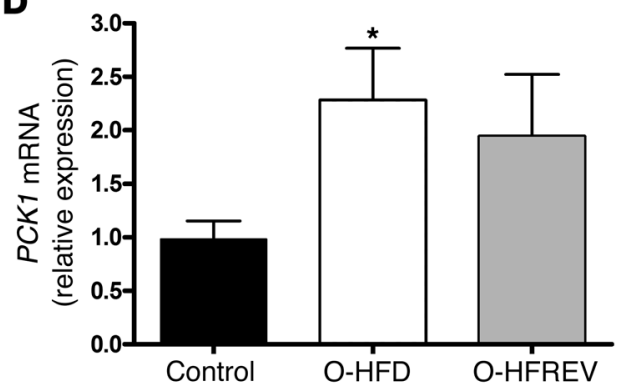

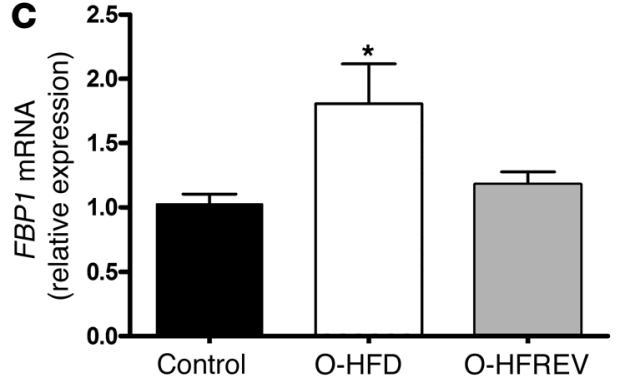

E

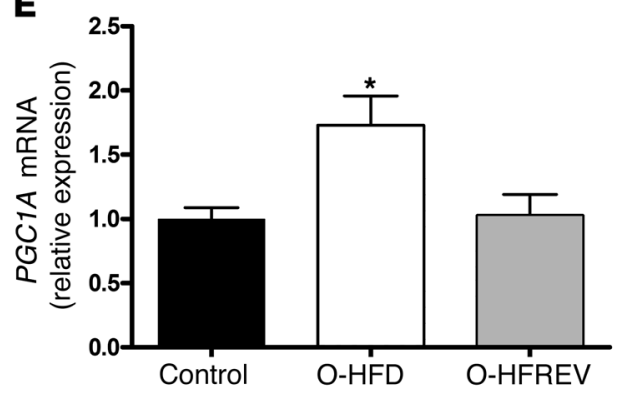

\section{Figure 5}

Maternal diet reversal partially prevents the development of fatty liver in the fetal offspring. (A) Liver TG levels for O-HFY4 and O-HFREV animals. Dashed line represents TG levels in normal control fetal offspring. ${ }^{\#} P<0.05$ versus $\mathrm{O}-\mathrm{HFY} 4$ (2-tailed Student's $t$ test). (B-E) Relative difference in G6P (B), FBP1 (C), PCK1 (D), and PGC1A (E) expression in the fetal liver of control $(n=9)$, O-HFD $(n=15)$, and O-HFREV $(n=7)$ groups. Data were obtained from samples obtained at different times and run in different assays, but were normalized to control samples that were present in every assay. O-HFD values represent the average of both O-HFD-R and O-HFD-S groups from Figure $4 .{ }^{*} P<0.05$ versus control (ANOVA with Bonferonni's multiple comparison test). There was no significant difference between the O-HFREV and either the control or the O-HFD groups. abundance or phosphorylation of the major components of the insulin signaling cascade, such as Akt, p85, and IRS1, or changes in several lipogenic transcription factors, such as PPAR $\gamma$, SREBP1C, and LXR (Supplemental Table 3).

Fetal hepatic steatosis is attenuated by a bealthy maternal diet. As described previously, 7 of the HFY4 females that became pregnant also became pregnant during the HFREV phase, thus allowing for a paired analysis between sequential fetuses. O-HFREV animals had body weights that were comparable to the control fetal offspring and slightly higher than their O-HFY4 siblings (Table 3); however, the difference was not significantly different. The O-HFREV group also had normal glycerol and total TG levels. Reflecting the changes in plasma lipids, O-HFREV animals had a $37 \%$ reduction in liver TG accumulation compared with O-HFY4 animals (Figure 5A); however, liver TG levels, although reduced, did not return to levels found in control fetuses from healthy mothers never exposed to an energy-dense HFD. Additionally, expression of gluconeogenic genes (G6P, FBP1, PCK1, and PGC1A mRNA) in the O-HFREV group was partially reversed, with values that were not significantly different from the control or O-HFD groups (Figure 5, B-E).

\section{Discussion}

The most notable finding of this study is that chronic maternal HFD consumption, independent of maternal obesity or diabetes, significantly increased the risk of NAFLD in the developing NHP fetus that persisted into the postnatal period. The evidence for NAFLD includes a 3-fold increase in liver TG levels, activation of several markers of oxidative stress, and premature activation of genes in the gluconeogenic pathway. It has been demonstrated in numerous models, including rodents, sheep, and NHPs, that manipulation of the maternal diet or hormone environment can affect the development of metabolic systems in the offspring (15-18, 22-24, 64). Using NHP models, Nathanielsz and colleagues have demonstrated a broad range of metabolic and developmental defects in the offspring of mothers with nutritional restriction (65-70). These broad effects are surprising, because the animals are nutritionally restricted by only $30 \%$. This suggests that primate pregnancies can be affected by subtle changes in nutrition. In the current studies, the animals were fed a diet that derived $35 \%$ of its calories from fat and was also high in total calories; this diet is similar to the typical American diet (71). Several studies in rodents have also investigated the effects of a maternal HFD on the development of metabolic abnormalities in the offspring $(21,24,72-79)$. While these studies describe a broad range of metabolic abnormalities, only a few describe increased TGs in the liver of adult offspring of pregnant rats fed a HFD; however, none of them determined whether the fatty liver develops prior to or after the development of obesity.

It is well recognized that maternal diabetes, obesity, and even glycemic control within ranges considered normal can markedly affect the developing fetus, thereby increasing the risk of metabolic dis- 


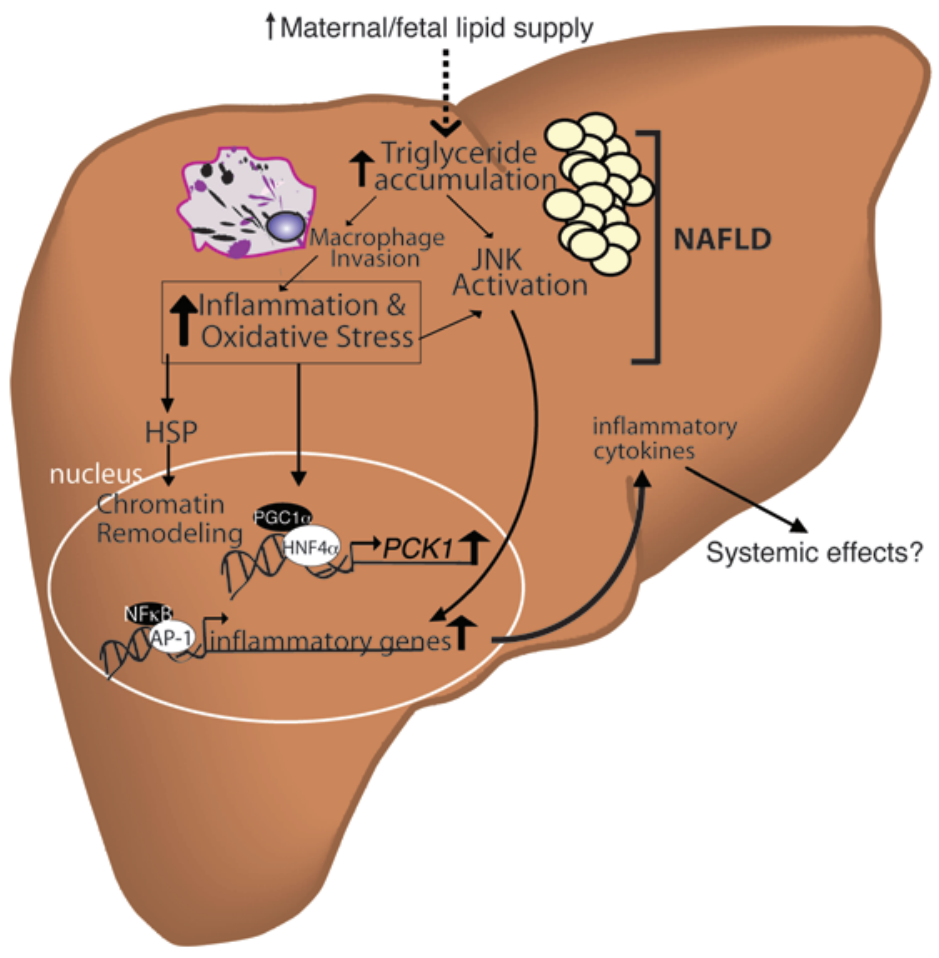

\section{Figure 6}

Effects of increased maternal/fetal lipid supply on hepatic lipotoxicity in the fetus. An increase in the availability of lipids to the fetus during early development prior to adipose tissue accretion results in lipid accumulation in nonadipose fetal tissues, leading to toxic effects. In O-HFD fetal liver, there was a significant increase in fetal hepatic TG accumulation that correlated with increased JNK activation. Liver staining revealed substantial elevations in markers of oxidative stress and damage in O-HFD livers. It is likely that the elevated TG levels lead to macrophage infiltration and contribute to the liver inflammation. Liver TG accumulation and inflammation are important events underlying NAFLD. The elevation in liver stress activates heat shock proteins (HSPs), which have previously been shown to be involved in chromatin remodeling (epigenetic programming; ref. 98). Oxidative stress and JNK activation triggers transcription for genes involved in gluconeogenesis as well as inflammation. Upregulation of inflammatory cytokines likely contributes to the progression of NAFLD, while early activation of gluconeogenic genes, such as PCK1, in fetal liver may predispose offspring for increased hepatic gluconeogenesis and insulin resistance. ease later in life $(7-11,25,80)$. One of the most important outcomes of this study was the fatty liver phenotype observed in the liver of O-HFD-S and O-HFD-R animals, which suggests that chronic HFD consumption may result in greater lipid transfer to the fetus regardless of maternal obesity. Because of the observational design of our study, we cannot conclude that maternal hyperlipidemia is the sole cause of the adverse outcomes observed; however, such a relationship is plausible. The strong correlation between maternal and fetal glycerol levels suggests that simply increasing maternal lipid as a source of calories in the diet can contribute to changes in the fetus, independent of adiposity or level of insulin resistance in the mother. The increase in liver TGs does not appear to be caused by an increase in de novo lipogenesis, as there was no change in mRNA or protein expression of any of the lipogenic enzymes analyzed (Supplemental Table 3). Indeed, the combination of increased lipid exposure, low fetal insulin, and no change in hepatic enzymes involved in lipogenesis suggests that excess lipid accumulation is a result of increased maternal lipid transfer. Although alterations in glucose metabolism are often considered the primary metabolic adaptations during pregnancy, substantial alterations occur in lipid metabolism as well. In human and rodent pregnancies, there is a shift toward increased lipolysis during the third trimester, and this is thought to be attributable, at least in part, to the normal insulin resistance that occurs during this period in pregnancy. Lipolysis from maternal adipose tissue increases plasma glycerol concentration and elevates fatty acids. Combined with fatty acids from dietary lipids and hepatic TGs, this leads to an increase in TG-rich lipoproteins in maternal circulation available for delivery to the placenta and ultimately provides an increased flux of fatty acids to the fetus (48-50). It is possible that insulin resistance in the HFD-fed NHPs may contribute to greater shunting of lipids to the fetus; however, evidence of marked NAFLD was observed in both the O-HFD-S and O-HFD-R animals. Interestingly, fetal liver TG levels were not significantly correlated with maternal or fetal circulating glycerol or TG levels. This suggests that there may be some intrahepatic mechanisms, such as reduced TG export, decreased FA oxidation, or other factors, that increase the risk for steatosis during fetal development. Further study is needed to identify such factors. Furthermore, we recognize that the HFD also has a higher caloric density and more saturated fat; thus it is not possible to definitively identify the major contributor - lipid or total calories - to the fetal lipotoxicity.

One hypothesis for the potential adverse effects of excess lipids on the fetal liver during development relates to the lack of WAT during the early third trimester $(36,37)$, which could be used to buffer the excess lipids. In adults, for example, diet-induced obesity overwhelms the WAT stores, and fat accumulates in ectopic organs, such as liver and skeletal muscle, causing insulin resistance. This is illustrated nicely in the "fatless mouse" models as well as in patients with lipodystrophy; in the latter, in the absence of WAT, fat is stored in the liver and muscle, leading to severe insulin resistance (38-45). With transplantation of wild-type WAT into this model, lipids dissipate from the liver and deposit in fat pads, and insulin resistance resolves. Therefore, it is possible that as WAT develops during the third trimester in the primate fetus, the fat in the liver will be mobilized and stored in the WAT. However, in O-HFD animals carried to full term and studied at P30 and P180, the fatty liver persisted; although the relative differences were notably less during the postnatal period than during the fetal period, the elevated hepatic TGs were accompanied by a 2 -fold increase in percent body fat in O-HFD. The persistence of the fatty liver may not be surprising, given that the offspring are kept with mothers who remain on the HFD and are concurrently breastfeeding. This reduction in liver TG levels could be caused by several factors in the postnatal period, including the development of WAT, but may also be caused by increased physical activity and fuel utilization 
of the animals as they become independent from their mothers. Juvenile monkeys are very active, especially in group or social housing. Further studies are needed to determine whether the fatty liver persists when O-HFD animals are switched to a low-fat diet during the postnatal period. Studies in rodents have shown that feeding a HFD to pregnant rats throughout pregnancy and during lactation causes a fatty liver phenotype in male offspring that persists into adulthood, even after animals return to a standard chow diet (79). These rats also displayed abnormalities in the insulin signaling cascade in the liver and were obese and insulin resistant; however, neither the gluconeogenic pathway nor the early development of fatty liver in the fetal or newborn pups was investigated.

One of the primary concerns about lipid accumulation within the liver is lipotoxicity, which can lead to insulin resistance, oxidative stress and/or damage, activation of proinflammatory cytokines, and ultimately liver fibrosis and permanent tissue damage (81-84). In the current study, we observed no hyperinsulinemia or any abnormalities in the insulin signaling cascade (either by gene expression or by protein phosphorylation); however, preliminary microarray studies and confirmatory PCR studies identified increased expression of several genes involved in the oxidative stress pathway and proinflammatory cytokines (D. Marks, unpublished observations). The activation of the oxidative stress pathway was confirmed by immunohistochemistry and immunoblot analysis. Furthermore, we also observed elevations in a wide range of inflammatory cytokines in fetal circulation (Supplemental Table 2). Our current understanding of the mechanisms responsible for the development of pediatric nonalcoholic steatohepatitis (NASH) remains limited. Whether this disorder has a fetal component as a result of the marking or "memory" of the liver during fetal life is as yet unknown. The data shown here suggest that the accumulation of excess lipids in the fetal liver is not benign and is associated with increased stress activation (p-JNK) and inflammatory cytokine gene activation, both pathophysiological features found in pediatric patients with NASH. Investigation of the consequences of these histological and biochemical changes on the offspring are underway and may lead to a better understanding of the pathophysiology of pediatric fatty liver disease.

An important question raised by our results and those of others is whether the fatty liver phenotype can be prevented by dietary intervention. In the current study, we chose a straightforward intervention of switching animals that had been on the HFD for more than 4 years (both HFD-S and HFD-R animals) to the low-fat standard chow diet specifically during the fifth-year pregnancy. During the diet reversal protocol, all animals showed a slight improvement of glucose tolerance; however, obese animals remained obese and were still substantially insulin resistant. In spite of this, there was marked improvement, but not complete normalization, in fetal hepatic TGs and partial normalization of the expression of the gluconeogenic enzymes in the O-HFREV group. These results suggest that simply changing to a normal low-fat diet, specifically during pregnancy, can lower, but not eliminate, the risk for fetal hepatic steatosis. Age, parity, or increased prepregnancy BMI could not account for the higher than normal fetal TG accumulation, as the control animals on the standard chow diet had similar numbers of pregnancies, and the effect of the diet reversal was similar in both O-HFREV-S and O-HFREV-R groups. These results imply that a chronic high-calorie, HFD could have long-term effects on maternal/fetal fuel metabolism in future pregnancies. The biological mechanisms through which this HFD during one pregnancy may modify the risk for fetal steatosis in subsequent pregnancies remains unclear. However, the failure of the diet reversal to completely normalize fetal hepatic TGs suggests that the supply or type of endogenous fatty acids may play an important role in addition to the maternal diet. It is well known that maternal obesity and even glucose in the normal range can have a substantial impact on fetal development and health; our current studies did not determine the relative contribution of maternal obesity to the fatty liver phenotype in the offspring. Nevertheless, there were major benefits associated with the diet reversal, which suggests that this is at least a reasonable strategy to help improve the metabolic health of the developing fetus.

Our results also demonstrate that chronic exposure to lipids during pregnancy triggered increased gluconeogenic gene expression associated with PGC1 $\alpha$ deacetylation and increased $\mathrm{HNF} 4 \alpha$ expression in the fetal liver, suggesting an important early molecular mechanism by which excess lipids may reprogram hepatic lipid and glucose metabolism in the fetus. The deacetylation of PGC1 $\alpha$ has been shown previously to increase PGC1 $\alpha$ activity to induce $P C K 1$ transcription and hepatic glucose output in transgenic mice $(61,62)$. PGC1 $\alpha$, along with increased $\mathrm{HNF} 4 \alpha$, is associated with increased PCK1 and G6P mRNA $(85,86)$, and may therefore play an important role in coordinating the premature induction of genes involved in gluconeogenesis that was observed in the O-HFD group. HNF $4 \alpha$ can be directly modulated by fatty acyl-CoA derivatives $(87,88)$, emphasizing its unique role in transcriptional regulation by nutrients and hormones. Moreover, HNF4 $\alpha$ can functionally bind to the promoters of up to $12 \%$ of all hepatic genes (89), suggesting that HNF $4 \alpha$ has broad activities and may contribute to a much larger regulatory network of essential metabolic processes in the liver. Although the increases in gluconeogenic gene expression at G130 were relatively small compared with expression in adult liver, in transgenic mice engineered to overexpress hepatic $P C K 1$ at birth, a 2-fold increase was sufficient to induce hepatic glucose production, insulin resistance, and impaired glucose tolerance in adults (69), suggesting that relatively small increases during development could prove to be significant in the evolution of diabetes. These data would be greatly strengthened by measures of increased hepatic glucose production; however, this was not technically feasible in the NHP fetus. While there could be evidence for greater gluconeogenesis after birth, most studies suggest that this evolves over time, and our animals are in the process of postweaning development. Further studies are currently underway to examine direct binding to the PCK1 promoter and to determine whether increased hepatic glucose production is present during the first year of life in these animals.

In utero adaptations, now referred to as fetal metabolic programming, were initially documented by Hales, Barker, and colleagues, who described increased cardiovascular mortality and impaired glucose tolerance in association with poor fetal growth (90). In recent years, the number of babies born large for gestational age has risen dramatically, and along with larger size comes greater risk for developing metabolic syndrome in adulthood $(12,13,19)$. Interestingly, these heavier babies are often born to mothers who are insulin resistant and obese, suggesting a crucial role for maternal overnutrition $(33,91,92)$. Here we illustrated an expanded concern for maternal HFD consumption and risks for NAFLD in offspring independent of maternal obesity or insulin resistance. While the fetuses from HFD-fed NHP mothers weighed slightly less than controls during the early third trimester, at P30 they were normal size and by P90, the animals were significantly fatter (but 
not heavier), emphasizing the importance of a shift in body composition imposed by early exposure to the HFD.

In summary, our data suggest that exposure to maternal lipidderived fuels during early pregnancy leads to an excess of lipid accumulation in the liver of the fetal and neonatal offspring (Figure 6). Lipid accumulation in the liver is associated with lipotoxicity, likely leading to macrophage infiltration and increased inflammatory cytokine production. This inflammation likely drives the activation of the oxidative stress pathway, which in turn can activate the transcriptional regulators of hepatic gluconeogenesis. Our group has previously demonstrated an increase in heat shock proteins, histone acetylation, and histone deacetylase activity in these fetal livers, suggesting long-term modifications in transcription (93). Furthermore, offspring had increased adiposity and liver TGs, which indicates that the fatty liver does persist beyond the development of WAT stores. Of great concern is our population of NHP mothers who were neither obese nor insulin resistant prior to pregnancy, but with exposure to a chronic HFD showed a fetal pattern of metabolic abnormalities identical to that of the fetuses from mothers with increased weight gain and insulin resistance. Although our study focused on the fetal liver, it is highly likely that the lipotoxicity is not limited to the fetal liver. Indeed, an increase in aortic fatty streaks has previously been observed in human fetuses from mothers with chronically or acutely elevated cholesterol, despite the lack of correlation between maternal and fetal cholesterol levels (94). Our findings of increased fetal steatosis and subsequent adiposity support what we believe to be a previously unrecognized risk of fetal programming of obesity resulting from early exposure to excess maternal lipids in utero. Finally, the current dietary guidelines for lowering blood glucose in pregnancy include substituting lipids for carbohydrates (95); however, the results of the current study emphasize the need to carefully balance carbohydrate and fat intake during pregnancy to reduce the risk of both hyperglycemia and hyperlipidemia during this critical developmental phase. Failure to recognize that maternal diet and maternal obesity play a critical role in fetal programming of adult disease may ultimately lead to an acceleration in the obesity epidemic through successive generations, independent of further genetic or environmental factors (96).

\section{Methods}

Experimental design. All animal procedures were in accordance with the guidelines of the Institutional Animal Care and Use Committee of the ONPRC. Age- and weight-matched young adult female Japanese macaques, 5-6 years of age at the start of the studies, were socially housed in indoor/ outdoor enclosures in groups of 5-9 with 1-2 males per group. Animals were separated into 2 groups: the control group was fed standard monkey chow that provides $14 \%$ of calories from fat (Monkey Diet no. 5052; Lab Diet), and the HFD group was fed a diet that supplied $32 \%$ of calories from fat (Custom Diet 5A1F; Test Diet) and included calorically dense treats (see Table 1). Both diets are sufficient in vitamin, mineral, and protein content for normal growth. Prior to this study, all animals were maintained on standard monkey chow in large outdoor enclosures and were naive to any experimental protocols.

Because these animals are socially housed, individual food intake and energy expenditure could not be measured. Bimonthly, body weights were measured and blood samples were taken for hormone analysis after an overnight fast. Animals were also checked for pregnancies by palpation and confirmed and aged by ultrasound. Twice a year, once during the nonbreeding season (August to September) and once during the third tri- mester of pregnancy (as determined by ultrasound), animals underwent an i.v. GTT to determine insulin sensitivity (see below). All of the above procedures were done under ketamine sedation $(5-10 \mathrm{mg} / \mathrm{kg})$.

The animals in the study were allowed to breed naturally. For the experiments involving fetuses, pregnancies were terminated by cesarean section, performed by ONPRC veterinarians, at G130 as determined by ultrasound. Both groups consisted of primiparous and multiparous pregnancy animals. All pregnancies were singleton. For the experiments involving postnatal offspring, the pregnancies were allowed to progress to natural birth. The offspring were maintained with their birth mothers on the same diet as consumed during pregnancy. To minimize stress during the perinatal period, offspring were not weighed until P30. In a preliminary study, a small cohort of animals was terminated at P30. In the follow-up study, offspring were weighed and body composition determined by DEXA at P30, P90, and P180. Liver biopsies were obtained by laparoscope at P180.

During the fifth year, HFREV animals were switched back to the standard monkey chow specifically during the breeding/pregnancy season (starting in October). The control group was maintained on the standard chow diet through this period.

Fetuses delivered from animals maintained on the control diet or HFD for 2-4 years were used. This time period correlates with changes in body weight in the HFD-S adult females. Immediately after cesarean section, the fetuses were delivered to necropsy, where they were weighed and measured. The fetuses were deeply anesthetized with sodium pentobarbital (>30 mg/ $\mathrm{kg}$ i.v.) and exsanguinated. Organs from these animals were quickly removed and weighed. The organs were dissected and either frozen in liquid nitrogen or fixed in $4 \%$ paraformaldehyde in phosphate buffer ( $\mathrm{pH} 7.4$ ).

Maternal insulin sensitivity. We performed i.v. GTTs twice a year on pregnant (early third trimester) and nonpregnant females after an overnight fast. Animals were sedated with ketamine $(10 \mathrm{mg} / \mathrm{kg})$ and administered a glucose bolus (50\% dextrose solution) at a dose of $0.6 \mathrm{~g} / \mathrm{kg}$ via the saphenous vein. Baseline blood samples were obtained prior to the infusion, and $1-\mathrm{ml}$ blood samples were taken at 1, 3, 5, 10, 20, 40, and 60 min after infusion via the femoral artery. Glucose was measured immediately using a OneTouch Ultra Blood Glucose Monitor (LifeScan), and the remainder of the blood was kept in heparinized tubes on ice for insulin measurement. After i.v. GTT, samples were centrifuged, and plasma was stored at $-80^{\circ} \mathrm{C}$ until assayed. Insulin was assayed in plasma by RIA (catalog no. RI-13K; Linco). HOMAIR was determined as previously described by Bonora et al. and Matthews et al. $(46,47)$, calculated as fasting serum insulin $(\mu \mathrm{U} / \mathrm{ml}) \times$ fasting plasma glucose $(\mathrm{mmol} / \mathrm{l}) / 22.5$.

Immunoblot analysis. Liver sections were homogenized in ice-cold lysis buffer containing $20 \mathrm{mM}$ Tris, pH 7.5; $150 \mathrm{mM} \mathrm{NaCl}$; 1\% Igepal; $20 \mathrm{mM}$ $\mathrm{NaF} ; 2 \mathrm{mM}$ EDTA; $2.5 \mathrm{mM}$ sodium pyrophosphate; $20 \mathrm{mM} \beta$-glycerophosphate; $10 \%$ glycerol; and inhibitors (Pefabloc and Mini-Complete protease inhibitor; Roche; and Phosphate Inhibitor cocktail 1/2; Sigma-Aldrich). Homogenates were solubilized for $1 \mathrm{~h}$ at $4{ }^{\circ} \mathrm{C}$ and then centrifuged at $12,000 \mathrm{~g}$ for $15 \mathrm{~min}$ to remove insoluble material. Protein concentration was determined using the BCA Protein Assay kit (Pierce). Nuclear extracts for PGC1 IP were prepared using a nuclear extraction kit (Pierce). For IP, anti-rabbit PGC1 $\alpha$ (Santa Cruz Biotechnology Inc.) was added to $100 \mu \mathrm{g}$ nuclear lysates and allowed to rotate overnight at $4{ }^{\circ} \mathrm{C}$. Protein A Sepharose beads (Amersham Biosciences) prewashed in PBS were added, rotated for $2 \mathrm{~h}$ at $4^{\circ} \mathrm{C}$, and then centrifuged at $12,000 \mathrm{~g}$. The antibody bead lysate was resuspended in $50 \mu \mathrm{l}$ Laemlli sample buffer and heated at $70^{\circ} \mathrm{C}$ for $10 \mathrm{~min}$. For immunoblot analysis, whole tissue homogenates $(50 \mu \mathrm{g})$ or nuclear IPs were run on 7\% Tris-Acetate precast gels (Invitrogen) and then transferred to polyvinylidene difluoride membranes (BioRad Laboratories). After transfer, membranes were exposed to the appropriate primary and secondary antibodies (BioRad Laboratories). The following antibodies were used: p-JNK 
(catalog no. 9251; Cell Signaling Technology), acetylated lysine (catalog no. 9441; Cell Signaling Technology), PGC1 $\alpha$ (catalog no. SC13067; Santa Cruz Biotechnology Inc.), HNF4 $\alpha$ (catalog no. SC6556; Santa Cruz Biotechnology Inc.), GAPDH (catalog no. SC20357; Santa Cruz Biotechnology Inc.), and JNK (catalog no. AF1387; R\&D Systems). Immunoreactive products were detected using Western Lightning enhanced chemiluminescence (Perkin Elmer) and exposure to X-OMAT sheet film (Eastman Kodak Co.). Protein levels were quantified using a FlurChem $Q$ analysis system (AlphaInnotech) and expressed relative to an internal standard run on every gel in duplicate. GAPDH was measured as a loading control.

$T G$ analysis. Liver TGs were measured by a modified Folch method that was described previously (97). Briefly, fetal livers were homogenized in 2:1 chloroform/methanol, and TGs were extracted overnight. The following day, organic and inorganic layers were separated by adding $0.6 \% \mathrm{NaCl}$, then the samples were thoroughly vortexed and centrifuged at $13,400 \mathrm{~g}$ at $4^{\circ} \mathrm{C}$ for $5 \mathrm{~min}$. The organic phase was carefully isolated and dried under nitrogen, and samples were resuspended in $200 \mu \mathrm{l}$ isopropanol. TG concentration was determined using the Serum Triglyceride Determination Kit (catalog no. TR0100; Sigma-Aldrich). An internal standard of $1 \mathrm{mM}$ tripalmitin was run during every extraction to calculate percent recovery.

RNA and quantitative RT-PCR. Total RNA was isolated from liver tissue derived from G130 fetuses from control diet- and HFD-fed mothers using TRIzOL (Invitrogen). mRNA was measured by real-time quantitative RT-PCR using a PRISM 7700 Sequences Detector System (Applied Biosystems). The following gene expression assays designed against human mRNA sequences were purchased from Applied Biosystems: PGC1A (assay no. Hs00173304_m1), PCK1 (assay no. Hs00159918_m1), G6P (assay no. Hs00978991_g1), and FBP1 (assay no. Hs00983318_m1).

Morphological analysis. Fresh-frozen liver was sectioned at $10 \mu \mathrm{m}$, fixed in $10 \%$ zinc formalin at $4{ }^{\circ} \mathrm{C}$ for $10 \mathrm{~min}$, and stained with Oil Red $\mathrm{O}$ and hematoxylin (American Master Tech Scientific Inc.). Liver tissue postfixed in $10 \%$ zinc formalin was embedded in paraffin and sectioned at $20 \mu \mathrm{m}$. The sections were deparaffinized with xylene and ethanol, blocked in $2 \%$ normal donkey serum, and incubated with mouse anti-HNE at 1:4 (catalog no. MHN-100P; Jaica) or goat anti-8-OH-DG diluted 1:500, followed by biotinylated donkey anti-mouse (catalog no. 715-066-150; Jackson) or donkey anti-goat (catalog no. 705-065-147; Jackson), respectively, both diluted 1:600. Sections were then incubated with Vectastain ABC Elite kit (catalog no. PK-6100; Vector Laboratories) followed by nickel DAB for 6 minutes. Finally, HNE sections were counterstained with hematoxylin for $1 \mathrm{~min}$, and 8-OH-DG sections were counterstained in neutral red for 30 seconds. For all histochemistry, a qualitative analysis was done by an observer blinded to the treatment group. The observer scaled the samples as having low, modest, or high staining.

Plasma hormone measurements. Blood samples were collected in heparinized Vacutainer tubes (BD) from the fetal aorta and centrifuged at $200 \mathrm{~g}$ for $20 \mathrm{~min}$.
Plasma insulin was measured using an in-house assay on a Roche Diagnostics Elecsys 2010 clinical assay platform, and leptin levels were measured using an RIA kit directed against human leptin (catalog no. PL-84K; Linco).

Proteome profiler cytokine array. Relative expression of plasma cytokines was quantified using the Proteome Profiler Human Cytokine Array, Panel A Array Kit (R\&D Systems). The array was performed according to the manufacturer's exact specifications using $250 \mu \mathrm{l}$ plasma ( $n=3$ [control diet]; 4 [HFD]). For maximum sensitivity of the assay, overnight incubation of blots with the plasma was used. ECL incubation was performed for $5 \mathrm{~min}$ using the SuperSignal West Femto Chemiluminescent Kit (Thermo Scientific Pierce), and then images were captured and analyzed using FluoroChem FC2 Imaging Illuminator coupled with AlphaEaseFC software (version 4.0; Alpha Innotech).

Statistics. Data are expressed as mean \pm SEM. For the maternal and fetal data, differences between the 3 groups (control, HFD-S, and HFD-R) were tested by 1-way ANOVA with Bonferroni post-hoc analysis. Animals were classified as HFD-S or HFD-R based on weight gain, response to i.v. GTT, and percent body fat change in the nonpregnant state, as described in Results. When comparable changes were observed in O-HFD-S and O-HFD-R groups, the 2 groups were combined, and a 2-tailed Student's $t$ test was used to compare values with offspring of control mothers. To investigate the effects of maternal diet reversal on gene expression, samples from the HFREV and control groups were run in the same assays and compared by 2-tailed Student's $t$ test. A $P$ value less than 0.05 was considered significant.

\section{Acknowledgments}

This work was funded by NIH grants DK-60685 (to K.L. Grove), DK-79194 (to K.L. Grove), RR00163 (to K.L. Grove and ONPRC), DKF32-075252 (to C.E. McCurdy), and DK-062155 (to J.E. Friedman). The authors would like to acknowledge the technical assistance of Lindsay Pranger, Sandra Joachim, Christopher Osman, Diana Takahashi, and the Division of Animal Resources and Veterinary staff at ONPRC for their technical assistance with these studies. The authors also acknowledge David Hess and the RIA Core.

Received for publication May 11, 2007, and accepted in revised form November 26, 2008.

Address correspondence to: Kevin L. Grove, Division of Neuroscience, ONPRC, Oregon Health and Science University, 505 NW 185th, Beaverton, Oregon 97007, USA. Phone: (503) 690-5380; Fax: (503) 690-5384; E-mail: grovek@ohsu.edu. Or to: Jacob E. Friedman, Department of Pediatrics, University of Colorado School of Medicine, Mail Stop 8106, PO Box 6511, Aurora, Colorado 80045, USA. Phone: (303) 724-3983; Fax: (303) 724-3920; E-mail: jed.friedman@ucdenver.edu.
1. Ogden, C.L., et al. 2006. Prevalence of overweight and obesity in the United States, 1999-2004. JAMA. 295:1549-1555.

2. Ogden, C.L., Flegal, K.M., Carroll, M.D., and Johnson, C.L. 2002. Prevalence and trends in overweight among US children and adolescents, 1999-2000. JAMA. 288:1728-1732.

3. Hedley, A.A., et al. 2004. Prevalence of overweight and obesity among US children, adolescents, and adults, 1999-2002. JAMA. 291:2847-2850.

4. Schwimmer, J.B., et al. 2005. Histopathology of pediatric nonalcoholic fatty liver disease. Hepatology. 42:641-649.

5. Patton, H.M., et al. 2006. Pediatric nonalcoholic fatty liver disease: a critical appraisal of current data and implications for future research. J. Pediatr. Gastroenterol. Nutr. 43:413-427.
6. Catalano, P.M., and Ehrenberg, H.M. 2006. The short- and long-term implications of maternal obesity on the mother and her offspring. BJOG. 113:1126-1133.

7. Gillman, M.W., Rifas-Shiman, S., Berkey, C.S., Field, A.E., and Colditz, G.A. 2003. Maternal gestational diabetes, birth weight, and adolescent obesity. Pediatrics. 111:e221-e226.

8. Lepercq, J., et al. 2001. Heterogeneity of fetal growth in type 1 diabetic pregnancy. Diabetes Metab. 27:339-344.

9. Lepercq, J., Hauguel-De Mouzon, S., Timsit, J., and Catalano, P.M. 2002. Fetal macrosomia and maternal weight gain during pregnancy. Diabetes Metab. 28:323-328.

10. Lindsay, R.S., et al. 2000. Type 2 diabetes and low birth weight: the role of paternal inheritance in the association of low birth weight and diabetes. Diabetes. 49:445-449.

11. Weyer, C., Pratley, R.E., Lindsay, R.S., and Tataranni, P.A. 2000. Relationship between birth weight and body composition, energy metabolism, and sympathetic nervous system activity later in life. Obes. Res. 8:559-565.

12. Dabelea, D., et al. 2000. Intrauterine exposure to diabetes conveys risks for type 2 diabetes and obesity: a study of discordant sibships. Diabetes. 49:2208-2211.

13. Dabelea, D., Knowler, W.C., and Pettitt, D.J. 2000. Effect of diabetes in pregnancy on offspring: follow-up research in the Pima Indians. J. Matern. Fetal Med. 9:83-88.

14. Grove, K.L., Grayson, B.E., Glavas, M.M., Xiao, X.Q., and Smith, M.S. 2005. Development of metabolic 
systems. Physiol. Behav. 86:646-660.

15. Xiao, X.Q., et al. 2007. Excess weight gain during the early postnatal period is associated with permanent reprogramming of brown adipose tissue adaptive thermogenesis. Endocrinology. 148:4150-4159.

16. McMillen, I.C., Adam, C.L., and Muhlhausler, B.S. 2005. Early origins of obesity: programming the appetite regulatory system. J. Physiol. 565:9-17.

17. McMillen, I.C., Muhlhausler, B.S., Duffield, J.A., and Yuen, B.S. 2004. Prenatal programming of postnatal obesity: fetal nutrition and the regulation of leptin synthesis and secretion before birth. Proc. Nutr. Soc. 63:405-412.

18. Muhlhausler, B.S., et al. 2005. Impact of glucose infusion on the structural and functional characteristics of adipose tissue and on hypothalamic gene expression for appetite regulatory neuropeptides in the sheep fetus during late gestation. J. Physiol. 565:185-195.

19. Silverman, B.L., Landsberg, L., and Metzger, B.E. 1993. Fetal hyperinsulinism in offspring of diabetic mothers. Association with the subsequent development of childhood obesity. Ann. N. Y. Acad. Sci. 699:36-45.

20. Salsberry, P.J., and Reagan, P.B. 2005. Dynamics of early childhood overweight. Pediatrics. 116:1329-1338

21. Armitage, J.A., Taylor, P.D., and Poston, L. 2005. Experimental models of developmental programming: consequences of exposure to an energy rich diet during development. J. Physiol. 565:3-8.

22. Sedova, L., et al. 2007. Sucrose feeding during pregnancy and lactation elicits distinct metabolic response in offspring of an inbred genetic model of metabolic syndrome. Am. J. Physiol. Endocrinol. Metab. 292:E1318-E1324.

23. Srinivasan, M., et al. 2006. Maternal high-fat diet consumption results in fetal malprogramming predisposing to the onset of metabolic syndrome-like phenotype in adulthood. Am. J. Physiol. Endocrinol. Metab. 291:E792-E799.

24. Srinivasan, M., et al. 2006. Maternal hyperinsulinemia predisposes rat fetuses for hyperinsulinemia, and adult-onset obesity and maternal mild food restriction reverses this phenotype. Am. J. Physiol. Endocrinol. Metab. 290:E129-E134.

25. Metzger, B.E., et al. 2008. Hyperglycemia and adverse pregnancy outcomes. N. Engl. J. Med. 358:1991-2002.

26. Di Cianni, G., et al. 2005. Maternal triglyceride levels and newborn weight in pregnant women with normal glucose tolerance. Diabet. Med. 22:21-25.

27. Kelishadi, R., Badiee, Z., and Adeli, K. 2007. Cord blood lipid profile and associated factors: baseline data of a birth cohort study. Paediatr. Perinat. Epidemiol. 21:518-524.

28. Gallou-Kabani, C., et al. 2007. Resistance to highfat diet in the female progeny of obese mice fed a control diet during the periconceptual, gestation, and lactation periods. Am. J. Physiol. Endocrinol. Metab. 292:E1095-E1100.

29. Khan, N.A. 2007. Role of lipids and fatty acids in macrosomic offspring of diabetic pregnancy. Cell Biochem. Biophys. 48:79-88.

30. Seppala-Lindroos, A., et al. 2002. Fat accumulation in the liver is associated with defects in insulin suppression of glucose production and serum free fatty acids independent of obesity in normal men. J. Clin. Endocrinol. Metab. 87:3023-3028.

31. McCarty, M.F. 2005. De novo synthesis of diacylglycerol in endothelium may mediate the association between PAI-1 and the insulin resistance syndrome. Med. Hypotheses. 64:388-393.

32. Knight, B., et al. 2007. The impact of maternal glycemia and obesity on early postnatal growth in a nondiabetic Caucasian population. Diabetes Care. 30:777-783.

33. Lawlor, D.A., et al. 2007. Epidemiologic evidence for the fetal overnutrition hypothesis: findings from the mater-university study of pregnancy and its outcomes. Am. J. Epidemiol. 165:418-424.

34. Taylor, G.M., Alexander, F.E., and D'Souza, S.W. 2006. Interactions between fetal HLA-DQ alleles and maternal smoking influence birthweight. Paediatr. Perinat. Epidemiol. 20:438-448.

35. Kral, J.G., et al. 2006. Large maternal weight loss from obesity surgery prevents transmission of obesity to children who were followed for 2 to 18 years. Pediatrics. 118:e1644-e1649.

36. Symonds, M.E., Mostyn, A., Pearce, S., Budge, H., and Stephenson, T. 2003. Endocrine and nutritional regulation of fetal adipose tissue development. J. Endocrinol. 179:293-299.

37. Stephenson, T., et al. 2001. Fetal and neonatal adipose maturation: a primary site of cytokine and cytokine-receptor action. Biochem. Soc. Trans. 29:80-85.

38. Kim, J.K., Gavrilova, O., Chen, Y., Reitman, M.L., and Shulman, G.I. 2000. Mechanism of insulin resistance in A-ZIP/F-1 fatless mice. J. Biol. Chem. 275:8456-8460

39. Kim, Y.B., Shulman, G.I., and Kahn, B.B. 2002. Fatty acid infusion selectively impairs insulin action on Akt1 and protein kinase C lambda /zeta but not on glycogen synthase kinase-3. J. Biol. Chem. 277:32915-32922.

40. Pajvani, U.B., et al. 2005. Fat apoptosis through targeted activation of caspase 8: a new mouse model of inducible and reversible lipoatrophy. Nat. Med. 11:797-803.

41. Trujillo, M.E., Pajvani, U.B., and Scherer, P.E. 2005. Apoptosis through targeted activation of caspase 8 ("ATTAC-mice"): novel mouse models of inducible and reversible tissue ablation. Cell Cycle. 4:1141-1145.

42. Agarwal, A.K., Barnes, R.I., and Garg, A. 2004. Genetic basis of congenital generalized lipodystrophy. Int. J. Obes. Relat. Metab. Disord. 28:336-339.

43. Chehab, F.F. 2008. Obesity and lipodystrophy - where do the circles intersect? Endocrinology. 149:925-934

44. Simha, V., and Garg, A. 2006. Lipodystrophy: lessons in lipid and energy metabolism. Curr. Opin. Lipidol. 17:162-169.

45. Unger, R.H. 2003. Minireview: weapons of lean body mass destruction: the role of ectopic lipids in the metabolic syndrome. Endocrinology. 144:5159-5165.

46. Bonora, E., et al. 2000. Homeostasis model assessment closely mirrors the glucose clamp technique in the assessment of insulin sensitivity: studies in subjects with various degrees of glucose tolerance and insulin sensitivity. Diabetes Care. 23:57-63.

47. Matthews, D.R., et al. 1985. Homeostasis model assessment: insulin resistance and beta-cell function from fasting plasma glucose and insulin concentrations in man. Diabetologia. 28:412-419.

48. Haggarty, P. 2002. Placental regulation of fatty acid delivery and its effect on fetal growth - a review. Placenta. 23(Suppl. A):S28-S38.

49. Herrera, E., and Amusquivar, E. 2000. Lipid metabolism in the fetus and the newborn. Diabetes Metab. Res. Rev. 16:202-210.

50. Herrera, E., Amusquivar, E., Lopez-Soldado, I., and Ortega, H. 2006. Maternal lipid metabolism and placental lipid transfer. Horm. Res. 65(Suppl. 3):59-64.

51. Jones, C.T., and Rolph, T.P. 1985. Metabolism during fetal life: a functional assessment of metabolic development. Physiol. Rev. 65:357-430.

52. Charlton, M. 2004. Nonalcoholic fatty liver disease: a review of current understanding and future impact. Clin. Gastroenterol. Hepatol. 2:1048-1058.

53. Pessayre, D. 2007. Role of mitochondria in nonalcoholic fatty liver disease. J. Gastroenterol. Hepatol. 22(Suppl. 1):S20-S27.
54. Yoshiuchi, K., et al. 2008. Direct monitoring of in vivo ER stress during the development of insulin resistance with ER stress-activated indicator transgenic mice. Biochem. Biophys. Res. Commun. 366:545-550.

55. Matsuzawa, N., et al. 2007. Lipid-induced oxidative stress causes steatohepatitis in mice fed an atherogenic diet. Hepatology. 46:1392-1403.

56. Vincent, H.K., and Taylor, A.G. 2006. Biomarkers and potential mechanisms of obesity-induced oxidant stress in humans. Int. J. Obes. (Lond.) 30:400-418.

57. Seki, S., et al. 2002. In situ detection of lipid peroxidation and oxidative DNA damage in non-alcoholic fatty liver diseases. J. Hepatol. 37:56-62.

58. Girard, J. 1986. Gluconeogenesis in late fetal and early neonatal life. Biol. Neonate. 50:237-258.

59. Bohme, H.J., Sparmann, G., and Hofmann, E. 1983. Biochemistry of liver development in the perinatal period. Experientia. 39:473-483.

60. McCurdy, C.E., and Friedman, J.E. 2006. Early foetal programming of hepatic gluconeogenesis: Glucocorticoids strike back. Diabetologia. 49:1138-1141.

61. Hanson, R.W., and Reshef, L. 1997. Regulation of phosphoenolpyruvate carboxykinase (GTP) gene expression. Annu. Rev. Biochem. 66:581-611.

62. Kalhan, S., and Parimi, P. 2000. Gluconeogenesis in the fetus and neonate. Semin. Perinatol. 24:94-106.

63. Rodgers, J.T., et al. 2005. Nutrient control of glucose homeostasis through a complex of PGC1alpha and SIRT1. Nature. 434:113-118.

64. Vuguin, P.M. 2007. Animal models for small for gestational age and fetal programming of adult disease. Horm. Res. 68:113-123.

65. Schlabritz-Loutsevitch, N.E., et al. 2007. Metabolic adjustments to moderate maternal nutrient restriction. Br. J. Nutr. 98:276-284.

66. Schlabritz-Loutsevitch, N., et al. 2007. Moderate maternal nutrient restriction, but not glucocorticoid administration, leads to placental morphological changes in the baboon (Papio sp.). Placenta. 28:783-793.

67. Nijland, M.J., et al. 2007. Non-human primate fetal kidney transcriptome analysis indicates mammalian target of rapamycin (mTOR) is a central nutrient-responsive pathway. J. Physiol. 579:643-656.

68. McDonald, T.J., Nijland, M.J., and Nathanielsz, P.W. 2007. The insulin-like growth factor system and the fetal brain: effects of poor maternal nutrition. Rev. Endocr. Metab. Disord. 8:71-84.

69. Li, C., et al. 2007. The IGF axis in baboon pregnancy: placental and systemic responses to feeding $70 \%$ global ad libitum diet. Placenta. 28:1200-1210.

70. Cox, L.A., et al. 2006. Effect of 30 per cent maternal nutrient restriction from 0.16 to 0.5 gestation on fetal baboon kidney gene expression. J. Physiol. 572:67-85.

71. U.S. Department of Agriculture, Agricultural Research Service. 2008. Nutrient intakes from food: mean amounts consumed per individual, one day, 2005-2006. http://www.ars.usda.gov/SP2UserFiles/ Place/12355000/pdf/0506/Table_1_NIF_05.pdf.

72. Cerf, M.E., et al. 2005. Islet cell response in the neonatal rat after exposure to a high-fat diet during pregnancy. Am. J. Physiol. Regul. Integr. Comp. Physiol. 288:R1122-R1128.

73. Cerf, M.E., Williams, K., Chapman, C.S., and Louw, J. 2007. Compromised beta-cell development and beta-cell dysfunction in weanling offspring from dams maintained on a high-fat diet during gestation. Pancreas. 34:347-353.

74. Cerf, M.E., Muller, C.J., Du Toit, D.F., Louw, J., and Wolfe-Coote, S.A. 2006. Hyperglycaemia and reduced glucokinase expression in weanling offspring from dams maintained on a high-fat diet. Br. J. Nutr. 95:391-396.

75. Bayol, S.A., Simbi, B.H., and Stickland, N.C. 2005. A maternal cafeteria diet during gestation and 
lactation promotes adiposity and impairs skeletal muscle development and metabolism in rat offspring at weaning. J. Physiol. 567:951-961.

76. Bayol, S.A., Farrington, S.J., and Stickland, N.C. 2007. A maternal 'junk food' diet in pregnancy and lactation promotes an exacerbated taste for 'junk food' and a greater propensity for obesity in rat offspring. Br. J. Nutr. 98:843-851.

77. Holemans, K., Caluwaerts, S., Poston, L., and Van Assche, F.A. 2004. Diet-induced obesity in the rat: a model for gestational diabetes mellitus. Am. J. Obstet. Gynecol. 190:858-865.

78. Caluwaerts, S., et al. 2007. Diet-induced obesity in gravid rats engenders early hyperadiposity in the offspring. Metabolism. 56:1431-1438.

79. Buckley, A.J., et al. 2005. Altered body composition and metabolism in the male offspring of high fatfed rats. Metabolism. 54:500-507.

80. Metzger, B.E. 2007. Long-term outcomes in mothers diagnosed with gestational diabetes mellitus and their offspring. Clin. Obstet. Gynecol. 50:972-979.

81. Leclercq, I.A. 2007. Pathogenesis of steatohepatitis: insights from the study of animal models. Acta Gastroenterol. Belg. 70:25-31.

82. Picardi, A., et al. 2006. Diabetes in chronic liver disease: from old concepts to new evidence. Diabetes Metab. Res. Rev. 22:274-283.

83. Schaffler, A., Scholmerich, J., and Buchler, C. 2005. Mechanisms of disease: adipocytokines and visceral adipose tissue - emerging role in nonalcoholic fatty liver disease. Nat. Clin. Pract. Gastroenterol. Hepatol. 2:273-280.

84. Medina, J., Fernandez-Salazar, L.I., Garcia-Buey, L., and Moreno-Otero, R. 2004. Approach to the pathogenesis and treatment of nonalcoholic steatohepatitis. Diabetes Care. 27:2057-2066.

85. Rhee, J., et al. 2003. Regulation of hepatic fasting response by PPARgamma coactivator-1alpha (PGC-1): requirement for hepatocyte nuclear factor 4alpha in gluconeogenesis. Proc. Natl. Acad. Sci. U. S. A. 100:4012-4017.

86. Puigserver, P., et al. 2003. Insulin-regulated hepatic gluconeogenesis through FOXO1-PGC-1alpha interaction. Nature. 423:550-555.

87. Jump, D.B., and Clarke, S.D. 1999. Regulation of gene expression by dietary fat. Annu. Rev. Nutr. 19:63-90.

88. Massillon, D., Arinze, I.J., Xu, C., and Bone, F. 2003. Regulation of glucose-6-phosphatase gene expression in cultured hepatocytes and H4IIE cells by short-chain fatty acids: role of hepatic nuclear factor-4alpha. J. Biol. Chem. 278:40694-40701.

89. Odom, D.T., et al. 2004. Control of pancreas and liver gene expression by HNF transcription factors. Science. 303:1378-1381.

90. Hales, C.N., et al. 1991. Fetal and infant growth and impaired glucose tolerance at age 64. BMJ. 303:1019-1022.

91. Forsum, E., Lof, M., Olausson, H., and Olhager, E. 2006. Maternal body composition in relation to infant birth weight and subcutaneous adipose tissue. Br. J. Nutr. 96:408-414.

92. Harvey, N.C., et al. 2007. Parental determinants of neonatal body composition. J. Clin. Endocrinol. Metab. 92:523-526.

93. Aagaard-Tillery, K.M., et al. 2008. Developmental origins of disease and determinants of chromatin structure: maternal diet modifies the primate fetal epigenome. J. Mol. Endocrinol. 41:91-102.

94. Napoli, C., et al. 1999. Influence of maternal hypercholesterolaemia during pregnancy on progression of early atherosclerotic lesions in childhood: Fate of Early Lesions in Children (FELIC) study. Lancet. 354:1234-1241.

95. Metzger, B.E., and Coustan, D.R. 1998. Summary and recommendations of the Fourth International Workshop-Conference on Gestational Diabetes Mellitus. The Organizing Committee. Diabetes Care. 21(Suppl 2):B161-B167.

96. Ebbeling, C.B., Pawlak, D.B., and Ludwig, D.S 2002. Childhood obesity: public-health crisis, common sense cure. Lancet. 360:473-482.

97. Bruce, C.R., et al. 2003. Muscle oxidative capacity is a better predictor of insulin sensitivity than lipid status. J. Clin. Endocrinol. Metab. 88:5444-5451.

98. Ruden, D.M., Xiao, L., Garfinkel, M.D., and Lu, X. 2005. Hsp90 and environmental impacts on epigenetic states: a model for the trans-generational effects of diethylstibesterol on uterine development and cancer. Hum. Mol. Genet. 14:R149-R155. 